\title{
Single-photon emission from InGaAs quantum dots grown on (111) GaAs
}

Cite as: Appl. Phys. Lett. 96, 093112 (2010); https://doi.org/10.1063/1.3337097

Submitted: 17 September 2009 - Accepted: 08 February 2010 • Published Online: 04 March 2010

Erik Stock, Till Warming, Irina Ostapenko, et al.

\section{ARTICLES YOU MAY BE INTERESTED IN}

On-demand generation of background-free single photons from a solid-state source Applied Physics Letters 112, 093106 (2018); https://doi.org/10.1063/1.5020038

Single photon emission from individual GaAs quantum dots

Applied Physics Letters 82, 2206 (2003); https://doi.org/10.1063/1.1563050

Invited Review Article: Single-photon sources and detectors

Review of Scientific Instruments 82, 071101 (2011); https://doi.org/10.1063/1.3610677

母 QBLOX

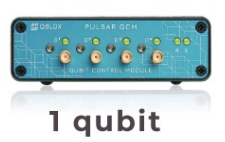

Shorten Setup Time Auto-Calibration More Qubits

Fully-integrated Quantum Control Stacks Ultrastable DC to $18.5 \mathrm{GHz}$ Synchronized $<<1 \mathrm{~ns}$ ultralow noise

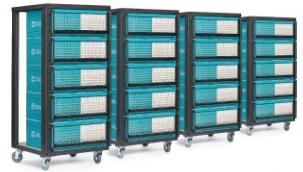

100 s qubits

visit our website > 


\section{Single-photon emission from InGaAs quantum dots grown on (111) GaAs}

Erik Stock, ${ }^{1, a)}$ Till Warming, ${ }^{1}$ Irina Ostapenko, ${ }^{1}$ Sven Rodt, ${ }^{1}$ Andrei Schliwa, ${ }^{1}$

Jan Amaru Töfflinger, ${ }^{1}$ Anatol Lochmann, ${ }^{1}$ Aleksandr I. Toropov, ${ }^{2}$ Sergej A. Moshchenko, ${ }^{2}$ Dimitry V. Dmitriev, ${ }^{2}$ Vladimir A. Haisler, ${ }^{2}$ and Dieter Bimberg ${ }^{1}$

${ }^{1}$ Institut für Festkörperphysik, Technische Universität Berlin, Hardenbergstrasse 36, 10623 Berlin, Germany

${ }^{2}$ Institute of Semiconductor Physics, Lavrenteva av 13, Novosibirsk 630090, Russia

(Received 17 September 2009; accepted 8 February 2010; published online 4 March 2010)

In this letter, we demonstrate that self-organized InGaAs quantum dots (QDs) grown on GaAs (111) substrate using droplet epitaxy have great potential for the generation of entangled photon pairs. The QDs show spectrally sharp luminescence lines and low spatial density. A second order correlation value of $\mathrm{g}^{(2)}(0)<0.3$ proves single-photon emission. By comparing the power dependence of the luminescence from a number of QDs we identify a typical luminescence fingerprint. In polarization dependent microphotoluminescence studies a fine-structure splitting ranging $\leq 40 \mu \mathrm{eV}$ down to the determination limit of our setup $(10 \mu \mathrm{eV})$ was observed. () 2010 American Institute of Physics. [doi:10.1063/1.3337097]

A network providing secure communication based on fundamental physical laws is possible if it uses quantum cryptography. The fundamental building block in such a quantum cryptography network is a robust and easy-tohandle source of entangled photon pairs. ${ }^{1}$

Self-organized quantum dots (QDs) (Ref. 2) are highly efficient electrically-driven single-photon sources. ${ }^{3-5}$ As proposed by Benson et al. ${ }^{6}$ a single QD will be an excellent source for entangled photon pairs if the fine-structure splitting (FSS) of the bright exciton states is reduced to less than the homogeneous line width.

For In(Ga)As QDs on (001) GaAs the FSS is nonzero, ${ }^{7-9}$ even for perfectly symmetric QDs, due to the asymmetric piezoelectric potential along the QDs base. For the first experimental demonstration of entangled photon pairs from QDs, postgrowth manipulation such as spectral filtering ${ }^{10}$ and magnetic fields ${ }^{11}$ were used. Other attempts to reduce the FSS include thermal annealing, ${ }^{12}$ external stress ${ }^{13}$ or electric fields. ${ }^{14}$ These techniques are complex and have to be adjusted for every single QD. Only if by accident the asymmetric potential becomes very small or is compensated by the QD elongation, the FSS will be small enough for the generation of entangled photons. ${ }^{15,16}$

In contrast to QDs on (001) GaAs, the piezoelectric field for QDs grown on (111) GaAs substrates is directed along the growth direction ${ }^{17}$ and does not lower the symmetry below $\mathrm{C}_{3 v}$ along the QDs base. It has been proposed recently that in this case the FSS is expected to be zero. ${ }^{5,18,19}$ Therefore triangular shaped InAs QDs grown on (111) GaAs surface might be ready-to-go sources for entangled photon pairs. $^{20}$

In previous attempts to grow InAs QDs on (111) GaAs substrates tetrahedral shaped recesses were used. ${ }^{21}$ This leads to the formation of rather large dots with an homogeneous line width of a few millielectron volts. Another attempt to grow QDs on (111) substrates ${ }^{22}$ resulted in a high density of very small QDs with a small localization energy, which are electronically close to quantum wells.

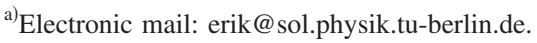

In this letter, we present the realization of self-organized InGaAs QDs on (111) GaAs substrates with low spatial density, sharp luminescence lines and a FSS close to or below our spectral resolution. These QDs are promising candidates for sources of entangled photon pairs.

The samples were grown by molecular beam epitaxy on GaAs (111) substrates having a miscut of $2^{\circ}$ in the [2 $\left.\overline{1} \overline{1}\right]$ direction, using a Riber32P system. On a $500 \mathrm{~nm}$ buffer layer a $50 \mathrm{~nm} \mathrm{Al} \mathrm{Al}_{0.6} \mathrm{Ga}_{0.4} \mathrm{As}$ layer followed by $65 \mathrm{~nm} \mathrm{GaAs}$ were deposited at $580{ }^{\circ} \mathrm{C}$. The QDs were grown using the droplet technique. ${ }^{23}$ The substrate temperature was reduced to $510{ }^{\circ} \mathrm{C}$ with closed As shutter. Nominally $1.5 \mathrm{ML}$ of Ga was deposited to saturate the excess surface As atoms and $\sim 2$ ML of In was deposited without As flux, forming In droplets. The formation of InGaAs QDs took place under As flux with closed In shutter and the rotation of the sample was switched off. The QD layer was capped with $65 \mathrm{~nm}$ GaAs followed by a second $\mathrm{Al}_{0.6} \mathrm{Ga}_{0.4}$ layer and a final $10 \mathrm{~nm}$ GaAs layer. The two AlGaAs layers prevent carrier diffusion from the QD layer to the bulk material. Atomic force images of uncapped QD layers show triangular shaped structures with a base length on the order of $100 \mathrm{~nm}$, while QD-sized structures could not be observed in these measurements.

The samples were excited with a frequency doubled $\mathrm{Nd}$ :YVO (yttrium vanadium oxide) laser $(532 \mathrm{~nm})$ in a microphotoluminescence setup at $15 \mathrm{~K}$, resulting in a spot diameter of $\sim 1 \mu \mathrm{m}$. The spectra were recorded with a monochromator and a Si-charge coupled device with a spectral resolution of $20 \mu \mathrm{eV}$. Using fitting procedures the energetic position of the luminescence can be determined within $10 \mu \mathrm{eV}$ precision. Polarization dependent measurements were performed using a fixed linear polarizer in front of the monochromator and a $\lambda / 2$ plate to rotate the polarization, making the polarization anisotropy of the monochromator negligible. The second-order correlation-function $g^{(2)}(\Delta t)$ was measured using a Hanbury-Brown-Twiss (HBT) setup, with a time resolution of $700 \mathrm{ps}$ and a spectral resolution of $0.3 \mathrm{meV}$.

Since the wafer rotation was switched off during growth, the luminescence of the samples varies significantly on different areas of the wafers. Some parts emit spectral broad 


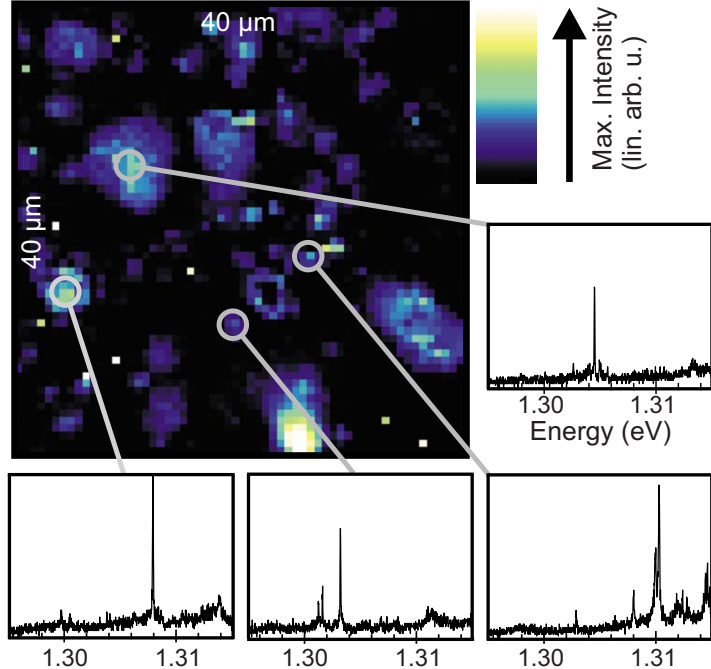

FIG. 1. (Color online) Spatial distribution of the QDs. The color scale indicates the value of the maximum intensity in the spectral range between 1.295 and $1.315 \mathrm{eV}$. The distance between dots emitting in this spectral range is on the order of micrometer. For four local spectra are plotted with the same scaling of the intensity.

luminescence, having little relation to QDs, whereas a small area showed spatially well separated luminescence centers, with spectral sharp luminescence lines. Figure 1 displays the spatial distribution of the luminescence in a spectral range from 1.295 to $1.315 \mathrm{eV}$. The color scale gives the value of the maximum intensity in this spectral range. On an area of $40 \times 40 \mu \mathrm{m}^{2}$ several luminescence centers are visible. The spectra of four of them are plotted with identical intensity scales around the frame. Sharp luminescence lines which are typical for the discrete energy levels of QDs are visible. The line width of most of them is limited by the spectral resolution of our setup and the intensity is comparable to the luminescence from a single QD grown on (001) substrate. The distance between two QDs can exceed a few micrometers and the spatial QD density is significantly less than $10^{9} \mathrm{~cm}^{-2}$. Thus single-QD spectroscopy without additional spatial selection is possible.

In order to check for the emission of single QDs we performed second-order correlation-function $\mathrm{g}^{(2)}(\Delta \mathrm{t})$ measurements with the HBT. Figure 2(a) shows the normalized $\mathrm{g}^{(2)}(\Delta \mathrm{t})$ function of one single luminescence line. The increased $\mathrm{g}^{(2)}$ at $\pm 5 \mathrm{~ns}$ is due to the so-called cross-talk. ${ }^{24}$ The measured value of $g^{(2)}(0)=0.3$ is mainly limited by the time resolution of the HBT. A simulation (black line), taking into account the time resolution of the setup, ${ }^{5}$ leads to a $\mathrm{g}^{(2)}(0)$ $=0.1$. Hence the emission of single photons and the existence of QDs is proven.

Next we want to investigate the electronic origin of the emission lines. Besides their shape the electronic structure of QDs grown on (111) GaAs is expected to differ substantially from that of (001) QDs. Consequently we will be facing "novel" emission patterns. We start our analysis by finding recurring luminescence line patterns in power-dependent measurements as characteristic fingerprints and follow up with the investigation of FSS in polarization-dependent measurements.

The upper part of Fig. 3 shows the excitation power dependence of several photoluminescence lines, originating from one QD. With increasing laser power three lumines-
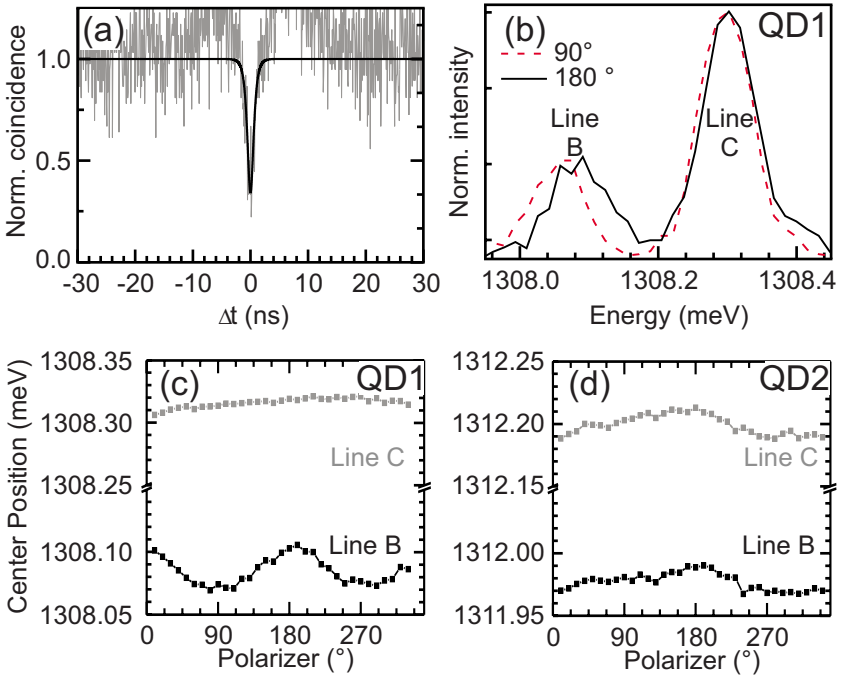

FIG. 2. (Color online) (a) Second-order correlation measurement (gray) and simulation (black) resulting in a $\mathrm{g}^{(2)}(0)$ value of 0.1 . (b) Spectra of QD1 with a polarizer at $90^{\circ}$ (red dashed) and $180^{\circ}$ (black solid), demonstrating the splitting of $40 \mu \mathrm{eV}$. (c) Spectral center position of the luminescence line $\mathrm{B}$ and $\mathrm{C}$ from QD1 obtained from a Gaussian fit in dependence of the polarization. (d) the same for QD2: no periodical dependence is visible indicating a vanishing FSS.

cence lines appear simultaneously. One intense line at 1.306 $\mathrm{eV}$ (in the following labeled as line A) and two lines at 1.308 $\mathrm{eV}$ separated by $300 \mu \mathrm{eV}$ (lines B and C). The intensity ratio of these lines is $4: 1: 2(\mathrm{~A}: \mathrm{B}: \mathrm{C})$ and their intensity depends almost linear on the excitation power. When the luminescence of these three lines saturates $($ at $\approx 100 \mathrm{nW}$ excitation power) several other lines appear and upon further increase in excitation power the lines A, B, and C fade. This scenery is typical for excitonic emission from a single QD. We therefore attribute the lines A, B, and C to single excitonic complexes of a single InAs QD grown on (111) GaAs substrate. For five more QDs we found a similar pattern of three lines with almost the same energetic distance and intensity ratio, exhibiting the same power dependence. In the following we will label all lines with this typical fingerprint by $\mathrm{A}, \mathrm{B}$, and $\mathrm{C}$.

The crucial parameter for the generation of entangled photon pairs is the FSS of the bright exciton state. In general,

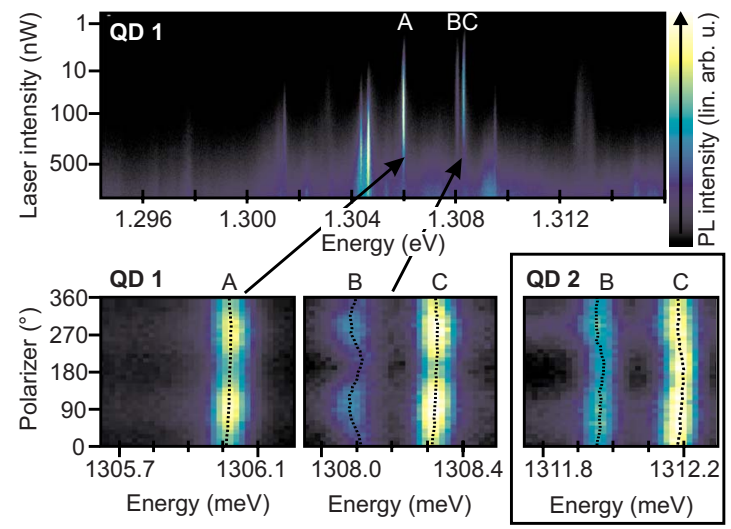

FIG. 3. (Color online) Top: excitation power dependence of the photoluminescence. The luminescence lines from single excitonic complexes are denoted by A, B, and C. Bottom: polarization dependence of the lines A, B, and $\mathrm{C}$ from QD1 and of lines B and C from QD2. The dotted lines show the center energy of the lines as in Fig. 2(c) and 2(d). 
several excitonic complexes can be observed in the luminescence of a single QD. The uncharged exciton (X) consisting of a single electron-hole pair, the negative/positive trion (two electrons/holes with a single hole/electron, $\mathrm{X}+, \mathrm{X}-$ ) or even multiply charged excitons $(\mathrm{X}++, \mathrm{X}-, \ldots){ }^{25}$ For nonzero FSS the bright exciton emission line will split into a linearly polarized doubled as will one of the emission lines of the $\mathrm{X}++{ }^{26}$ Therefore the FSS can be measured in the luminescence of the uncharged and of the doubly charged exciton.

In order to determine a possible FSS for QDs grown on (111) GaAs substrate we performed polarization dependent measurements on lines A, B, and C (bottom of Fig. 3). The intensities of lines $\mathrm{A}$ and $\mathrm{C}$ are clearly polarization dependent. For an angle of $90^{\circ}$ the intensity is 1.5 times larger than that for $180^{\circ}$, while the energetic position does not change. For QD1 line B on the other hand shows almost the same intensity for all polarizations but the energetic position (dashed line in Fig. 3) shifts. This is more visible in Fig. 2(b), which shows the spectra of QD1 for $90^{\circ}$ (red) and $180^{\circ}$ (black). The center positions of lines $\mathrm{B}$ and $\mathrm{C}$ obtained from a Gaussion fit for all polarization directions are plotted in Fig. 2(c). For line B a clear energetic change of about $40 \mu \mathrm{eV}$ with a periodicity of $180^{\circ}$ is clearly visible, whereas line $\mathrm{C}$ shows no systematic energetic shift. Therefore the luminescence of line B gives access to the FSS and originates either from the $\mathrm{X}$ or $\mathrm{X}++$. Line $\mathrm{A}$ and $\mathrm{C}$ are probably from trions. Spectroscopy on single QDs grown on (111) GaAs substrate is a new field and a complete assignment of the observed luminescence lines to transitions from well identified few-particle states requires further investigation like microphotoluminescence excitation spectroscopy ${ }^{27}$ and has to be addressed in future work.

For QD2 we found the same luminescence fingerprint of three lines. The polarization dependence of the lines B and C is shown in the right bottom part of Fig. 3 as a color plot and the energetic positions in Fig. 2(d). For QD2 line B exhibits only a very small energetic shift on the order of the determination limit of our setup $(10 \mu \mathrm{eV})$. In contrast to QD1 the spectral shift is not periodically and not reproducible. The FSS of QD1 was the largest we found and on five other QDs (not shown) it is ranging from $30 \mu \mathrm{eV}$ down or below the determination limit.

We attribute the nonzero FSS to two effects mainly. The QDs were grown on a (111) substrate with a miscut of $2^{\circ}$. This may lead to the growth of elongated QDs along the steps on the (111) surface. Second, even the growth of GaAs bulk material on this substrate orientation is challenging. The samples exhibit a high density of dislocations, which may influence the built-in piezoelectric field and cause strain in one preferential direction. This may result in an asymmetric confinement potential, similar to that of QDs on (001) substrate. By optimizing the growth process theses effects can be eliminated.

In conclusion we demonstrate the realization of selforganized InGaAs QDs on (111) GaAs substrates. The QDs have a very low spatial density and exhibit spectra with sharp and intense lines. The luminescence of the QDs demonstrates clear antibunching with an $\mathrm{g}^{(2)}(0)<0.3$ which proves single photon emission. On the basis of excitation power-dependent measurements on a number of QDs we were able to identify a typical luminescence fingerprint of QDs grown on (111) GaAs. One of these lines allows the measurement of the FSS. The observed FSS ranges from $40 \mu \mathrm{eV}$ down to the determination limit of our setup $(10 \mu \mathrm{eV})$. Our results present an important step toward the realization of a compact source of entangled photon pairs based on semiconductor technology.

This work was supported by Deutsche Forschungsgemeinschaft in the frame of SFB 787.

${ }^{1}$ N. Gisin, G. Ribordy, W. Tittel, and H. Zbinden, Rev. Mod. Phys. 74, 145 (2002).

${ }^{2}$ D. Bimberg, Electron. Lett. 44, 390 (2008).

${ }^{3}$ Z. Yuan, B. E. Kardynal, R. M. Stevenson, A. J. Shields, C. J. Lobo, K Cooper, N. S. Beattie, D. A. Ritchie, and M. Pepper, Science 295, 102 (2002).

${ }^{4}$ A. J. Shields, Nat. Photonics 1, 215 (2007).

${ }^{5}$ D. Bimberg, E. Stock, A. Lochmann, A. Schliwa, J. A. Töfflinger, W. Unrau, M. Münnix, S. Rodt, V. A. Haisler, A. I. Toropov, A. Bakarov, and A. K. Kalagin, IEEE Photonics Journal 1, 58 (2009).

${ }^{6}$ O. Benson, C. Santori, M. Pelton, and Y. Yamamoto, Phys. Rev. Lett. 84, 2513 (2000).

${ }^{7}$ V. D. Kulakovskii, G. Bacher, R. Weigand, T. Kummell, A. Forchel, E. Borovitskaya, K. Leonardi, and D. Hommel, Phys. Rev. Lett. 82, 1780 (1999).

${ }^{8}$ C. Santori, D. Fattal, M. Pelton, G. S. Solomon, and Y. Yamamoto, Phys. Rev. B 66, 045308 (2002).

${ }^{9}$ S. M. Ulrich, S. Strauf, P. Michler, G. Bacher, and A. Forchel, Appl. Phys. Lett. 83, 1848 (2003).

${ }^{10}$ N. Akopian, N. H. Lindner, E. Poem, Y. Berlatzky, J. Avron, D. Gershoni, B. D. Gerardot, and P. M. Petroff, Phys. Rev. Lett. 96, 130501 (2006).

${ }^{11}$ R. M. Stevenson, R. J. Young, P. Atkinson, K. Cooper, D. A. Ritchie, and A. J. Shields, Nature (London) 439, 179 (2006).

${ }^{12}$ R. Seguin, A. Schliwa, T. D. Germann, S. Rodt, K. Pötschke, A. Strittmatter, U. W. Pohl, D. Bimberg, M. Winkelnkemper, T. Hammerschmidt, and P. Kratzer, Appl. Phys. Lett. 89, 263109 (2006).

${ }^{13}$ S. Seidl, M. Kroner, A. Hogele, K. Karrai, R. J. Warburton, A. Badolato, and P. M. Petroff, Appl. Phys. Lett. 88, 203113 (2006).

${ }^{14}$ K. Kowalik, O. Krebs, A. Lemaitre, S. Laurent, P. Senellart, P. Voisin, and J. A. Gaj, Appl. Phys. Lett. 86, 041907 (2005).

${ }^{15}$ R. Seguin, A. Schliwa, S. Rodt, K. Pötschke, U. W. Pohl, and D. Bimberg, Phys. Rev. Lett. 95, 257402 (2005).

${ }^{16}$ R. Hafenbrak, S. M. Ulrich, P. Michler, L. Wang, A. Rastelli, and O. G. Schmidt, New J. Phys. 9, 315 (2007).

${ }^{17}$ M. Povolotskyi, A. Di Carlo, and S. Birner, Phys. Status Solidi C 1, 1511 (2004).

${ }^{18}$ A. Schliwa, M. Winkelnkemper, A. Lochmann, E. Stock, and D. Bimberg, Phys. Rev. B 80, 161307 (2009).

${ }^{19}$ R. Singh and G. Bester, Phys. Rev. Lett. 103, 063601 (2009).

${ }^{20}$ A. Schliwa, M. Winkelnkemper, and D. Bimberg, German Patent Application No. 102008036400.2 .

${ }^{21}$ Y. Sugiyama, Y. Sakuma, S. Muto, and N. Yokoyama, Appl. Phys. Lett. 67, 256 (1995).

${ }^{22}$ F. Y. Tsai and C. P. Lee, J. Appl. Phys. 84, 2624 (1998).

${ }^{23}$ T. Chikyow and N. Koguchi, Jpn. J. Appl. Phys., Part 2 29, L2093 (1990).

${ }^{24}$ During the detection of a photon on one avalanche photodiode (APD) a photon may be emitted by this APD. The photon can be detected by the second APD, resulting in a coincidence within a fixed time delay.

${ }^{25}$ R. J. Warburton, C. S. Durr, K. Karrai, J. P. Kotthaus, G. MedeirosRibeiro, and P. M. Petroff, Phys. Rev. Lett. 79, 5282 (1997).

${ }^{26}$ M. Ediger, G. Bester, B. D. Gerardot, A. Badolato, P. M. Petroff, K. Karrai, A. Zunger, and R. J. Warburton, Phys. Rev. Lett. 98, 036808 (2007).

${ }^{27}$ T. Warming, E. Siebert, A. Schliwa, E. Stock, R. Zimmermann, and D Bimberg, Phys. Rev. B 79, 125316 (2009). 